\title{
The Spin Waves in the Double Exchange Interaction Systems
}

\author{
Chong Der Hu \\ Department of Physics, National Taiwan University, Taipei, Taiwan, R.O.C.
}

(Received October 7, 1999)

\begin{abstract}
We performed a detailed study of the double exchange interaction. Having rearranged Kubo and Ohata's Hamiltonian [J. Phys. Soc. Jpn. 33 (1972) 21], we found that it can be treated perturbatively for the temperaure lower than $T_{\mathrm{C}}$. The spin wave propagator was calculated and the spin stiffness was computed. It agrees reasonablly well with experimental results. We believe that the double exchange interaction is the fundamental mechanism in the perovskite manganites.
\end{abstract}

KEYWORDS: colossal magnetoresistance, mangnites, double exchange

\section{$\S 1 . \quad$ Introduction}

In the past few years, we have seen a revival of the study of the perovskite manganites which had been known to have the so called colossal magnetoresistance phenomenum (CMR) long time ago. ${ }^{1,2)}$ The representative compound is $L a_{1-x} A_{x} M n O_{3}$ where $A$ represents a divalent element. The rich physics of these kind of compounds has induced earnest and sustained research. ${ }^{3)}$ Owing to different doping concentration $x$, the conduction electron density and the valence and the magnetic moment of mangnese ions vary. Combined with lattice distortion and temperature effect, these compounds exhibit many phases under different conditions, such as antiferromagnetic insulator, ferromagnetic insulator, ferromagnetic metal, charge ordering and paramagnetic insulator. The most prominent feature is the coupling between magnetic phase transition and the metal-insulator transition. Zener ${ }^{4)}$ had proposed an intuitively appealing mechanism, the double exchange interaction (DE). With the middle oxygen ion acting as medium, the conduction electrons can hop from a $\mathrm{Mn}^{+3}$ ion to a $\mathrm{Mn}^{+4}$ ion. Due to the on-site Coulomb repulsion, the electron spin must be parallel to the spins of Mn ions (Hund's rules) and thus creating magnetic coupling. This theory is able to explain the couplig between magnetic and transport properties since the ferromagnetic order favors the motions of conduction electrons. However, Millis et al.5) argued that the only enegy scale in DE is at least an order in magnitude larger than $T_{\mathrm{C}}$. Their calculation using the infinite-dimension Kondo lattice model also showed that $\mathrm{DE}$ is inadequate to explain the behavior of resistivity near $T_{\mathrm{C}}$. They proposed that the Jahn-Tellet effect should be considered. There are also experimental evidences indicating that the electron-lattice interaction is important. For example, the isotope effect on the thermoelectric power ${ }^{6)}$ and on $T_{\mathrm{C}} \cdot{ }^{7}$ A possibly related issue is the evidence of polaron formation. ${ }^{8}$ Thus raised question of whether DE is the most important mechanism in perovskite mangnites. We think it is.

Since it was proposed by Zener, DE had been fur- ther developed by Anderson and Hasegawa ${ }^{9)}$ and De Gennes. ${ }^{10)}$ Next major progress was made by Kubo and Ohata $^{11)}$ who used projection operators to project out the lowest-energy states according to Hund's rules and thus, simplified the Hamiltonian. It is not until the recently we have seen many theoretical works. ${ }^{12-18)}$ From our point of view, it is worthy to analyze DE carefully and make a detailed calculation to see its implication. Then the results can be a base to incoporate the JahnTeller effect. Certainly, as said by Müller-Hartmann and Dagotto, ${ }^{19)}$ it is crucial to have a proper quantum mechanical treatment of the spin system. Therefore, we developed a fully quantum mechanical calculation for DE. We chose to calculate the spin wave propagator at finite temperature because DE is closely related to the magnetic properties of the system and there are experimental data available. ${ }^{20,21)}$ The analysis of the Hamiltonian is in $\S 2$ and the calculation is in $\S 3$. Comparison with experiments and conclusion are in $\S 4$.

\section{§. Hamiltonian}

In the CMR manganites, the lowest state of a $\mathrm{Mn}^{+3}$ ion is the $S=2$ state for the total spin of four d-electrons, according to the Hund's rules. The $S=1$ configuration has a higher energy, by as much as $I=3 \mathrm{eV}$, as shown in band-structure calculations. ${ }^{22)}$ On the other hand, the band width is around $2.4 \mathrm{eV}$ which gives a hopping energy (to be denoted by $t$ ) of $0.2 \mathrm{eV}$. Therefore, the energy difference between the two spin configurations, $I$, is the largest energy scale. As we are interested in the spin waves, the low-lying excitations, we can restrict ourselves to the lower spin configuration. By doing this we can work in the large $I$ limit and simplify the Hamiltonian. The price to pay is that we have neglected the processes of the order $t / I$, such as an electron hopping between $\mathrm{Mn}^{+3}(S=1)$ and $\mathrm{Mn}^{+4}$ sites. Thus, we use the Hamiltonian developed by Kubo and Ohata: ${ }^{11}$

$$
H=-t \sum_{i, j, \alpha, \beta} c_{i \alpha}^{\dagger}\left(P_{i} P_{j}\right)_{\alpha \beta} c_{j \beta}
$$

Here and in all the following equations $i$ and $j$ are nearest 
neighbors. Considering the effect of the on-site exchange interaction, the projection operator $P$ is used to projects out the states in which the spins of holes anti-parallel to the local spins:

$$
P_{i}=\frac{S-\boldsymbol{S}_{i} \cdot \boldsymbol{\sigma}}{2 S+1}
$$

Within the Hamiltonian, the manganese ions can be $\mathrm{Mn}^{+4}$, which has spin $3 / 2$, or $\mathrm{Mn}^{+3}$ which has spin $S=2$. At a $\mathrm{Mn}^{+4}$ site, there is a hole whose spin is set to be antiparallel to the local spin $S=2$ by the projection operator. Equation (1) describes the hoppings of the holes between $\mathrm{Mn}^{+3}$ and $\mathrm{Mn}^{+4}$ sites. At finite temperatures, the spins of manganese ions are not always aligned, hence, there exist spin-up and spin-down holes. In the process of hopping, the spins of holes can be flipped though the total spin is conserved.

The Hamiltonian can be expanded as

$$
\begin{aligned}
H= & \frac{-t}{(2 S+1)^{2}} \sum_{i, j, \alpha, \beta} c_{i \alpha}^{\dagger}\left[S^{2}+\boldsymbol{S}_{i} \cdot \boldsymbol{S}_{j}\right. \\
& -\left(S S_{i z}+S S_{j z}-\frac{1}{2} S_{i-} S_{j+}+\frac{1}{2} S_{i+} S_{j-}\right) \sigma_{z} \\
& -\left(S S_{i-}+S S_{j-}-S_{i z} S_{j-}+S_{i-} S_{j z}\right) \frac{\sigma_{+}}{2} \\
& \left.-\left(S S_{i+}+S S_{j+}+S_{i z} S_{j+}-S_{i+} S_{j z}\right) \frac{\sigma_{-}}{2}\right]_{\alpha \beta} c_{j \beta} .
\end{aligned}
$$

It seems that there is only one energy scale, namely $t$. (The energy scale of the spin wave is $\left.t /(2 S+1) .{ }^{11)}\right)$ However, there are small parameters $x$ and $1 /(2 S+1)$. Because of the former, we consider only the terms with one fermion loop. To show that the latter exists, we write the Hamiltonian in the following way:

$$
H=H_{0}+H_{1}+H_{2}+H_{3}+H_{4}
$$

where

$$
\begin{aligned}
& H_{0}=-\frac{t}{N(2 S+1)^{2}} \sum_{i, j, \boldsymbol{k}, \sigma} \mathrm{e}^{\mathrm{i} \boldsymbol{k} \cdot \boldsymbol{R}_{i j}}\left[\left(S-\sigma\left\langle S_{z}\right\rangle\right)^{2} c_{\boldsymbol{k}, \sigma}^{\dagger} c_{\boldsymbol{k}, \sigma}+\left(S_{i z}+S_{j z}-2\left\langle S_{z}\right\rangle\right)\left(\left\langle S_{z}\right\rangle-\sigma S\right) n_{\boldsymbol{k}, \sigma}\right], \\
& H_{1}=-\frac{t}{N(2 S+1)^{2}} \sum_{i, j, \boldsymbol{k}, \boldsymbol{q}, \sigma} \mathrm{e}^{\mathrm{i} \boldsymbol{k} \cdot \boldsymbol{R}_{i j}+\mathrm{i} \boldsymbol{q} \cdot \boldsymbol{R}_{i}}\left(c_{\boldsymbol{k}-\boldsymbol{q}, \sigma}^{\dagger} c_{\boldsymbol{k}, \sigma}-n_{\boldsymbol{k}, \sigma}\right)\left(S_{i z}+S_{j z}-2\left\langle S_{z}\right\rangle\right)\left(\left\langle S_{z}\right\rangle-\sigma S\right), \\
& H_{2}=-\frac{t}{N(2 S+1)^{2}} \sum_{i, j, \boldsymbol{k}, \boldsymbol{q}, \sigma} \mathrm{e}^{\mathrm{i} \boldsymbol{k} \cdot \boldsymbol{R}_{i j}+\mathrm{i} \boldsymbol{q} \cdot \boldsymbol{R}_{i}} c_{\boldsymbol{k}-\boldsymbol{q}, \sigma}^{\dagger} c_{\boldsymbol{k}, \sigma}\left(S_{i z}-\left\langle S_{z}\right\rangle\right)\left(S_{j z}-\left\langle S_{z}\right\rangle\right), \\
& H_{3}=-\frac{t}{N(2 S+1)^{2}} \sum_{i, j, \boldsymbol{k}, \boldsymbol{q}, \sigma} \mathrm{e}^{\mathrm{i} \boldsymbol{k} \cdot \boldsymbol{R}_{i j}+\mathrm{i} \boldsymbol{q} \cdot \boldsymbol{R}_{i}}\left(c_{\boldsymbol{k}-\boldsymbol{q}, \sigma}^{\dagger} c_{\boldsymbol{k}, \sigma} S_{i-} S_{j+}+c_{\boldsymbol{k}-\boldsymbol{q}, \sigma}^{\dagger} c_{\boldsymbol{k}, \sigma} S_{i+} S_{j-}\right)
\end{aligned}
$$

and

$$
\begin{aligned}
H_{4}= & -\frac{t}{N(2 S+1)^{2}} \sum_{i, j, \boldsymbol{k}, \boldsymbol{q}} \mathrm{e}^{\mathrm{i} \boldsymbol{k} \cdot \boldsymbol{R}_{i j}+\mathrm{i} \boldsymbol{q} \cdot \boldsymbol{R}_{i}}\left[c_{\boldsymbol{k}-\boldsymbol{q}, \uparrow}^{\dagger} c_{\boldsymbol{k}, \downarrow}\left(S_{i z} S_{i-}-S_{i-} S_{j z}-S S_{i-}-S S_{j-}\right)\right. \\
& \left.+c_{\boldsymbol{k}-\boldsymbol{q}, \downarrow}^{\dagger} c_{\boldsymbol{k}, \uparrow}\left(-S S_{i+}-S S_{j+}+S_{i+} S_{j z}-S_{i z} S_{j+}\right)\right],
\end{aligned}
$$

where $\langle X\rangle$ is the thermal average of $X$ in the ensemble of $H_{0}, n_{\boldsymbol{k} \sigma}=\left\langle c_{\boldsymbol{k} \sigma}^{\dagger} c_{\boldsymbol{k} \sigma}\right\rangle$ and $N$ is the number of lattice sites. $H_{0}$ is the mean-field part of eq. (1). $H_{1}$ and $H_{2}$ represents the contribution of the quantum fluctuations of $S_{z} . H_{3}$ has the flipping of the local spins and $H_{4}$ is the hole spin-flip terms.

Since $H_{0}$ contains the most important contribution, we elaborated it in more details. First of all, the spin-up holes and spin-down holes form two bands. Assuming $\left\langle S_{z}\right\rangle>0$, the spin-down holes are the majority carriers. They have a smaller effective mass (proportional to $\left.\left(S+\left\langle S_{z}\right\rangle\right)^{-2}\right)$ and lower energy band bottom. The spinup holes are immobile, have zero energy at $T=0$, and $\left\langle S_{z}\right\rangle=S$. But they form a narrow band at low temperature. The bottom of the band is high compared to that of spin-down holes. Hence there are fewer of them. However, as temperature rises, $\left\langle S_{z}\right\rangle$ becomes smaller and two bands move closer to each other. The local spins have discrete enegy levels whose spacings are related to the hole energy and abundance. This is our basic picture.

The energy of an hole with spin polarization $\sigma$ is

$$
\varepsilon_{\boldsymbol{k}, \sigma}=-\left(S-\sigma\left\langle S_{z}\right\rangle\right)^{2} \varepsilon_{\boldsymbol{k}} .
$$

where

$$
\varepsilon_{\boldsymbol{k}}=\frac{t}{(2 S+1)^{2}} \sum_{n . n .} \mathrm{e}^{\mathrm{i} \boldsymbol{k} \cdot \boldsymbol{R}}
$$

As usual, $\sum_{n . n}$ sums over nearest neighbors and $\sigma= \pm 1$. If the local spins have $\left\langle S_{z}\right\rangle>0$, then the spin-down holes have lower energy. $H_{0}$ can be solved to give the zeroth order spin wave propagator. This will be given in next section.

It seems that $H_{1-4}$ are of the order $S^{0}$. Acutaly, their higher order terms are not. For example, the thermal average of the terms first order in $H_{1}$ vanish. The second order terms are proportional to

$$
\frac{t^{2}}{(2 S+1)^{4}}\left(\left\langle S_{z}^{2}\right\rangle-\left\langle S_{z}\right\rangle^{2}\right)\left(S_{z}-\sigma S\right)^{2} .
$$

It vanishes in the low temperature limit. At finite temperature, the criterion of our $1 /(2 S+1)$ expansion is

$$
\left\langle S_{z}^{2}\right\rangle-\left\langle S_{z}\right\rangle^{2} \leq S
$$

Using the Weiss' mean field theory with spin level spacing $\omega_{0}$, this is valid for $k_{\mathrm{B}} T \leq S \omega_{0}$. Thus, for $H_{1}$, we can have a $1 /(2 S+1)$ expansion except for the region near $T_{\mathrm{C}}$. 
Similar arguement can be applied to higher order terms and terms with $H_{2} . H_{3}$ and $H_{4}$ are the spin-flip terms. Any physical quantity involving spin-flipping must contain the factor $\left\langle S_{+} S_{-}\right\rangle$. It will be shown in next section that $\left\langle S_{+} S_{-}\right\rangle$is proportional to $S_{z}$. Thus, the terms second order in $H_{3}$ or $H_{4}$ are of the order $1 /(2 S+1)$. Therefore, $H_{1}, H_{2}, H_{3}$ and $H_{4}$ can be treated perturbatively. Due to the complexity of the Hamiltonian, we consider only the $O(1 /(2 S+1))$ terms.

\section{§3. Spin Wave Propagator}

We give some details of how to calculate the spin wave propagator in this section. The spin wave in the DE systems had been studied by Furukawa. ${ }^{23)}$ The reason why we embark on this subject is that we are interested in the finite temperature behavior. Readers will also notice that our approaches are different. In order to investigate the behavior of the spin waves, we calculate the spin wave propagator. Since we are interested in the finite temperature behavior of spin waves, those simplifications such as Holstein-Primakoff transformation will not be applied. Instead, we perform calculation with the expansion of $1 /(2 S+1)$. The leading term in $H$ is $H_{0}$. It is solved exactly. As a result, the zeroth order spin propagator $-\left\langle\widehat{T} S_{+}(\tau) S_{-}\left(\tau^{\prime}\right)\right\rangle$ where $\widehat{T}$ is the time-ordered operator, can be evaluated.

$$
\begin{aligned}
D_{0}\left(\mathrm{i} q_{n}\right) & =-\frac{1}{2} \int_{-\beta}^{\beta} \mathrm{d} \tau\left\langle\widehat{T} S_{+}(\tau) S_{-}\left(\tau^{\prime}\right)\right\rangle \mathrm{e}^{\mathrm{i} q_{n}\left(\tau-\tau^{\prime}\right)} \\
& =\frac{2\left\langle S_{z}\right\rangle}{\mathrm{i} q_{n}-\omega_{0}}
\end{aligned}
$$

where

$$
\omega_{0}=\frac{2}{N} \sum_{\boldsymbol{k}, \sigma} f_{\boldsymbol{k} \sigma}\left(\left\langle S_{z}\right\rangle-\sigma S\right) \varepsilon_{\boldsymbol{k}},
$$

comes from the commutator $H_{0}$ and $S_{+}$:

$$
\left[H_{0}, S_{+}\right]=-\omega_{0} S_{+} .
$$

and $f_{k_{\sigma}}$ is the Fermi-Dirac distribution function of holes with spin polarization $\sigma$. The derivation of eq. (12) was done by Giovannini et al. ${ }^{24)}$ We briefly outline its process. In view of eq. (14), we found

$$
S_{ \pm}(\tau)=\mathrm{e}^{\mp \tau \omega_{0}} S_{ \pm}
$$

and

$$
\begin{aligned}
D_{0}\left(\mathrm{i} q_{n}\right)= & -\sum_{l, m} \frac{\mathrm{e}^{-\beta E_{l}}}{2\left(\mathrm{i} q_{n}-\omega_{0}\right)}\left[\left(\mathrm{e}^{-\beta \omega_{0}}-1\right)\left|\left(S_{-}\right)_{m l}\right|^{2}\right. \\
& \left.+\left(1-\mathrm{e}^{\beta \omega_{0}}\right)\left|\left(S_{+}\right)_{m l}\right|^{2}\right]
\end{aligned}
$$

Since $E_{m}=E_{l}+\omega_{0}$, we reached eq. (12).

In the calculation of higher order terms, we confronted the complexity that the Wick's theorem is not applicable to spin operators. ${ }^{24)}$ As a result, there will be other propagators such as $\left\langle\widehat{T} S_{i+}(\tau) S_{j-}\left(\tau^{\prime}\right) S_{m z}\left(\tau_{1}\right)\right\rangle$, $\left\langle\widehat{T} S_{i+}(\tau) S_{j-}\left(\tau^{\prime}\right) S_{m z}\left(\tau_{1}\right) S_{l z}\left(\tau_{2}\right)\right\rangle$ and $\left\langle\widehat{T} S_{i+}(\tau) S_{j-}\left(\tau^{\prime}\right)\right.$ $\left.S_{m+}\left(\tau_{1}\right) S_{l-}\left(\tau_{2}\right)\right\rangle$. (The time arguement of $S_{z}$ is not irrelevant even though $\left[H_{0}, S_{z}\right]=0$. It helps determine the order of spin operators.) They can also be calculated $e x$ actly in the ensemble of $H_{0}$. The way to calculate these propagators is make contractions so that the final form contains only $S_{z} \cdot{ }^{24)}$ If this goal is reached we can evaluate the thermal average because $S_{z}$ and $H_{0}$ are commute. The procedure of computing $\left\langle\widehat{T} S_{+}(\tau) S_{-}\left(\tau^{\prime}\right) S_{z}\left(\tau_{1}\right)\right\rangle$ will be given as an example. Others will follow accordingly. We begin with the following identity:

$$
\begin{aligned}
& \left\langle\widehat{T} S_{i+}(\tau) S_{j-}\left(\tau^{\prime}\right) S_{m z}\left(\tau_{1}\right)\right\rangle \\
& =\delta_{i j}\left[\left(1-\delta_{i m}\right)\left\langle\widehat{T} S_{+}(\tau) S_{-}\left(\tau^{\prime}\right)\right\rangle\left\langle S_{z}\right\rangle\right. \\
& \left.\quad+\delta_{i m}\left\langle\widehat{T} S_{+}(\tau) S_{-}\left(\tau^{\prime}\right) S_{z}\left(\tau_{1}\right)\right\rangle\right]
\end{aligned}
$$

The meaning of eq. (17) is that if $S_{m z}$ operates on the same site as $S_{+} S_{-}$, then these three operators cannot be decoupled. Consider $\theta\left(\tau-\tau^{\prime}\right) \theta\left(\tau^{\prime}-\tau_{1}\right)\left\langle S_{+}(\tau) S_{-}\left(\tau^{\prime}\right) S_{z}\right\rangle$ (the order of spin operators is fixed). It belongs to the second term of eq. (17). One moves $S_{+}$in a cyclic fashion $^{25)}$ and creats various commutators. Once it was moved back to its original position, an additional factor of $\exp \left(-\beta \omega_{0}\right)$ was created from its commutator with $\exp \left(-\beta H_{0}\right)$ from the ensemble average. Thus, we found (using eq. (15))

$$
\begin{aligned}
& \theta(\tau-\left.\tau^{\prime}\right) \theta\left(\tau^{\prime}-\tau_{1}\right)\left\langle S_{+}(\tau) S_{-}\left(\tau^{\prime}\right) S_{z}\right\rangle \\
&= \theta\left(\tau-\tau^{\prime}\right) \theta\left(\tau^{\prime}-\tau_{1}\right) \mathrm{e}^{\omega_{0}\left(\tau^{\prime}-\tau\right)} \\
& \quad \times\left[\frac{2\left\langle S_{z}^{2}\right\rangle}{1-\mathrm{e}^{-\beta \omega_{0}}}-\frac{2 \mathrm{e}^{-\beta \omega_{0}}\left\langle S_{z}\right\rangle}{\left(1-\mathrm{e}^{-\beta \omega_{0}}\right)^{2}}\right]
\end{aligned}
$$

This way, we found that

$$
\begin{aligned}
\left\langle\widehat{T} S_{+}\right. & \left.(\tau) S_{-}\left(\tau^{\prime}\right) S_{z}\left(\tau_{1}\right)\right\rangle \\
= & -\mathcal{D}\left(\tau-\tau^{\prime}\right)\left\langle S_{z}^{2}\right\rangle-2\left\langle S_{z}\right\rangle \mathrm{e}^{\omega_{0}\left(\tau^{\prime}-\tau\right)}\left[n_{B}\left(n_{B}+1\right)\right. \\
& -n_{B} \theta\left(\tau-\tau_{1}\right) \theta\left(\tau_{1}-\tau^{\prime}\right) \\
& \left.+\left(n_{B}+1\right) \theta\left(\tau^{\prime}-\tau_{1}\right) \theta\left(\tau_{1}-\tau\right)\right]
\end{aligned}
$$

where

$$
\begin{aligned}
& \mathcal{D}\left(\tau_{1}-\tau_{2}\right) \\
& \quad=-2\left[n_{B} \theta\left(\tau_{2}-\tau_{1}\right)+\left(n_{B}+1\right) \theta\left(\tau_{1}-\tau_{2}\right)\right] \mathrm{e}^{\omega_{0}\left(\tau_{2}-\tau_{1}\right)}
\end{aligned}
$$

and

$$
n_{B}=\frac{1}{\mathrm{e}^{\beta \omega_{0}}-1} .
$$

We can calculate $\left\langle\widehat{T} S_{+}(\tau) S_{-}\left(\tau^{\prime}\right) S_{z}\left(\tau_{1}\right) S_{z}\left(\tau_{2}\right)\right\rangle$ and $\left\langle\widehat{T} S_{+}(\tau) S_{-}\left(\tau^{\prime}\right) S_{+}\left(\tau_{1}\right) S_{-}\left(\tau_{2}\right)\right\rangle$ the similar way. The results are given in Appendix A.

In general, the form of the spin wave propagator is

$$
D\left(\mathrm{i} q_{n}, \boldsymbol{q}\right)=\frac{2\left\langle S_{z}\right\rangle}{\mathrm{i} q_{n}-\omega_{0}-\Pi\left(\mathrm{i} q_{n}, \boldsymbol{q}\right)} .
$$

where $\Pi\left(\mathrm{i} q_{n}, \boldsymbol{q}\right)$ is the self-energy. In a perturbative series, we might get the form

$$
\begin{aligned}
& D\left(\mathrm{i} q_{n}, \boldsymbol{q}\right) \\
& \quad \simeq \frac{2\left\langle S_{z}\right\rangle}{\mathrm{i} q_{n}-\omega_{0}}-\frac{2\left\langle S_{z}\right\rangle Z^{\prime}\left(\mathrm{i} q_{n}\right)}{\mathrm{i} q_{n}-\omega_{0}}+\frac{2\left\langle S_{z}\right\rangle A\left(i q_{n}, \boldsymbol{q}\right)}{\left(\mathrm{i} q_{n}-\omega_{0}\right)^{2}}+\cdots
\end{aligned}
$$

This implies a spin wave propagator of the form 


$$
D\left(\mathrm{i} q_{n}, \boldsymbol{q}\right) \simeq \frac{2\left\langle S_{z}\right\rangle\left[1-Z^{\prime}\left(\mathrm{i} q_{n}\right)\right]}{\mathrm{i} q_{n}-\omega_{0}-A\left(\mathrm{i} q_{n}, \boldsymbol{q}\right)} .
$$

$1-Z^{\prime}\left(\mathrm{i} q_{n}\right)$ is the weight of the coherent part of the spin wave and $A\left(\mathrm{i} \omega_{0}, \boldsymbol{q}\right)$ gives the spin wave disper- sion relation and dissipation. We shall calculate $A\left(\mathrm{i} q_{n}, \boldsymbol{q}\right)$ and $Z^{\prime}\left(\mathrm{i} q_{n}\right)$ and retain only the terms of the order of $1 /(2 S+1)$.

In general, the full spin propagator is given by

$$
\begin{aligned}
D\left(\mathrm{i} q_{n}, \boldsymbol{q}\right)= & \frac{-1}{2 N} \int_{-\beta}^{\beta} \mathrm{d}\left(\tau-\tau^{\prime}\right) \sum_{i, j} \mathrm{e}^{\mathrm{i} q_{n}\left(\tau-\tau^{\prime}\right)+\mathrm{i} \boldsymbol{q} \cdot \boldsymbol{R}_{i j}} \\
& \times\left\langle\widehat{T} \exp \left\{-\int_{0}^{\beta} \mathrm{d} \tau_{1}\left[H_{1}\left(\tau_{1}\right)+H_{2}\left(\tau_{1}\right)+H_{3}\left(\tau_{1}\right)+H_{4}\left(\tau_{1}\right)\right]\right\} S_{i+}(\tau) S_{j-}\left(\tau^{\prime}\right)\right\rangle .
\end{aligned}
$$

Pertubatively, we have

$$
\begin{aligned}
& \left\langle\widehat{T} \exp \left\{-\int_{0}^{\beta} \mathrm{d} \tau_{1}\left[H_{1}\left(\tau_{1}\right)+H_{2}\left(\tau_{1}\right)+H_{3}\left(\tau_{1}\right)+H_{4}\left(\tau_{1}\right)\right]\right\} S_{i+}(\tau) S_{j-}\left(\tau^{\prime}\right)\right\rangle \\
& \simeq\left\langle\widehat{T} S_{i+}(\tau) S_{j-}\left(\tau^{\prime}\right)\right\rangle-\left\langle\widehat{T} S_{i+}(\tau) S_{j-}\left(\tau^{\prime}\right) \int_{0}^{\beta} \mathrm{d} \tau_{1}\left[H_{1}\left(\tau_{1}\right)+H_{2}\left(\tau_{1}\right)+H_{3}\left(\tau_{1}\right)+H_{4}\left(\tau_{1}\right)\right]\right\rangle \\
& \quad+\frac{1}{2}\left\langle\widehat{T} S_{i+}(\tau) S_{j-}\left(\tau^{\prime}\right) \int_{0}^{\beta} \mathrm{d} \tau_{1}\left[H_{1}\left(\tau_{1}\right)+H_{2}\left(\tau_{1}\right)+H_{3}\left(\tau_{1}\right)+H_{4}\left(\tau_{1}\right)\right] \cdot \int_{0}^{\beta} \mathrm{d} \tau_{2}\left[H_{1}\left(\tau_{2}\right)+H_{2}\left(\tau_{2}\right)+H_{3}\left(\tau_{2}\right)+H_{4}\left(\tau_{2}\right)\right]\right\rangle .
\end{aligned}
$$

Only $H_{3}$ contributes to the first order term:

$$
\sum_{i, j .} \mathrm{e}^{\mathrm{i} \boldsymbol{q} \cdot \boldsymbol{R}_{i j}}\left\langle\widehat{T} S_{i+}(\tau) S_{j-}\left(\tau^{\prime}\right) H_{3}\left(\tau_{1}\right)\right\rangle=-\left\langle\widehat{T} S_{+}(\tau) S_{-}\left(\tau_{1}\right)\right\rangle\left\langle\widehat{T} S_{+}\left(\tau_{1}\right) S_{-}\left(\tau^{\prime}\right)\right\rangle \frac{1}{N} \sum_{\boldsymbol{k}, \sigma} f_{\boldsymbol{k}, \sigma} \varepsilon_{\boldsymbol{k}-\boldsymbol{q}}
$$

The expressions of the second-order terms are quite lengthy. We have

$$
\begin{aligned}
& \sum_{i, j .} \mathrm{e}^{\mathrm{i} \boldsymbol{q} \cdot \boldsymbol{R}_{i j}}\left\langle\widehat{T} S_{i+}(\tau) S_{j-}\left(\tau^{\prime}\right) H_{1}\left(\tau_{1}\right) H_{1}\left(\tau_{2}\right)\right\rangle \\
& =\frac{1}{N} \sum_{\boldsymbol{p}, \sigma, l} \mathrm{e}^{\mathrm{i} p_{l}\left(\tau_{1}-\tau_{2}\right)} \chi_{z}\left(\mathrm{i} p_{l}, \boldsymbol{p}, \sigma\right)\left(\left\langle S_{z}\right\rangle-\sigma S\right)^{2}\left[\left\langle\widehat{T} S_{+}(\tau) S_{-}\left(\tau^{\prime}\right) S_{z}\left(\tau_{1}\right) S_{z}\left(\tau_{2}\right)\right\rangle-2\left\langle S_{z}\right\rangle\left\langle\widehat{T} S_{+}(\tau) S_{-}\left(\tau^{\prime}\right) S_{z}\left(\tau_{1}\right)\right\rangle\right. \\
& \left.\quad+\left\langle S_{z}\right\rangle^{2}\left\langle\widehat{T} S_{+}(\tau) S_{-}\left(\tau^{\prime}\right)\right\rangle+\left(\left\langle S_{z}^{2}\right\rangle-\left\langle S_{z}\right\rangle^{2}\right)\left\langle\widehat{T} S_{+}(\tau) S_{-}\left(\tau^{\prime}\right)\right\rangle\right]
\end{aligned}
$$

and

$$
\begin{aligned}
\sum_{i, j .} & \mathrm{e}^{\mathrm{i} \boldsymbol{q} \cdot \boldsymbol{R}_{i j}}\left\langle\widehat{T} S_{i+}(\tau) S_{j-}\left(\tau^{\prime}\right) H_{4}\left(\tau_{1}\right) H_{4}\left(\tau_{2}\right)\right\rangle \\
= & \sum_{\sigma, l} \mathrm{e}^{\mathrm{i} p_{l}\left(\tau_{1}-\tau_{2}\right)} \chi_{t}\left(\mathrm{i} p_{l}, \boldsymbol{q}, \sigma\right)\left\{\left\langle\widehat{T} S_{+}(\tau) S_{-}\left(\tau_{1}\right)\right\rangle\left\langle\widehat{T} S_{+}\left(\tau_{2}\right) S_{-}\left(\tau^{\prime}\right)\right\rangle+\left[\left\langle\widehat{T} S_{+}(\tau) S_{-}\left(\tau^{\prime}\right) S_{+}\left(\tau_{2}\right) S_{-}(\tau)\right\rangle\right.\right. \\
& \left.\left.-\left\langle\widehat{T} S_{+}(\tau) S_{-}\left(\tau_{1}\right)\right\rangle\left\langle\widehat{T} S_{+}\left(\tau_{2}\right) S_{-}\left(\tau^{\prime}\right)\right\rangle+\left\langle\widehat{T} S_{+}(\tau) S_{-}\left(\tau^{\prime}\right)\right\rangle\left\langle\widehat{T} S_{+}\left(\tau_{2}\right) S_{-}\left(\tau_{1}\right)\right\rangle\right]\right\}
\end{aligned}
$$

where

$$
\chi_{z}\left(\mathrm{i} p_{l}, \boldsymbol{p}, \sigma\right)=\frac{1}{N} \sum_{\boldsymbol{k}}\left(\varepsilon_{\boldsymbol{k}}+\varepsilon_{\boldsymbol{k}-\boldsymbol{p}}\right)^{2} \frac{f_{\boldsymbol{k}, \sigma}-f_{\boldsymbol{k}-\boldsymbol{p}, \sigma}}{\mathrm{i} p_{l}-\varepsilon_{\boldsymbol{k}, \sigma}+\varepsilon_{\boldsymbol{k}-\boldsymbol{p}, \sigma}}
$$

and

$$
\begin{aligned}
\chi_{t}\left(\mathrm{i} p_{l}, \boldsymbol{p}, \sigma\right)= & \frac{1}{N} \sum_{\boldsymbol{k}}\left[\left(S-\sigma\left\langle S_{z}\right\rangle\right) \varepsilon_{\boldsymbol{k}}+\left(S+\sigma\left\langle S_{z}\right\rangle\right) \varepsilon_{\boldsymbol{k}-\boldsymbol{p}}\right]^{2} \\
& \times \frac{f_{\boldsymbol{k}, \sigma}-f_{\boldsymbol{k}-\boldsymbol{p},-\sigma}}{\mathrm{i} p_{l}-\varepsilon_{\boldsymbol{k}, \sigma}+\varepsilon_{\boldsymbol{k}-\boldsymbol{p},-\sigma}}
\end{aligned}
$$

come from the hole polarization. The last terms of eqs. (27) and (28) give disconnected diagrams. They will be canceled by the corresponding diagrams in the thermodynamic potential. The term second order in $H_{2}$ is higher order in $1 /(2 S+1)$ except for

the region near $T_{\mathrm{C}}$, and can be neglected. The term $\left\langle\widehat{T} S_{i+}(\tau) S_{j-}\left(\tau^{\prime}\right) H_{1}\left(\tau_{1}\right) H_{2}\left(\tau_{2}\right)\right\rangle$ are also negligible for the similar reason. Hence, there are only three terms, namely, those in eqs. (26), (27) and (28) need to be considered. Among them, the spin-flip processes in eqs. (26) and (28) give $A\left(\mathrm{i} q_{n}, \boldsymbol{q}\right)$ and the $S_{z}$ related eq. (27) gives $Z^{\prime}\left(\mathrm{i} q_{n}\right)$. The full expressions are in Appedix A. Here, we retaining only the leading terms in eqs. $(\mathrm{A} \cdot 2)$ and $(\mathrm{A} \cdot 3)$,

$$
\begin{aligned}
Z^{\prime}\left(\mathrm{i} q_{n}\right)= & \frac{\beta}{2 N\left\langle S_{z}\right\rangle}\left[\left\langle S_{z}^{3}\right\rangle-2\left\langle S_{z}^{2}\right\rangle\left\langle S_{z}\right\rangle+\left\langle S_{z}\right\rangle^{3}\right] \\
& \times \sum_{\boldsymbol{p}, \sigma}\left(S-\sigma S_{z}\right)^{2} \chi_{z}(0, \boldsymbol{p}, \sigma),
\end{aligned}
$$

and 


$$
A\left(\mathrm{i} q_{n}, \boldsymbol{q}\right)=-\frac{2\left\langle S_{z}\right\rangle}{N} \sum_{\boldsymbol{k}, \sigma} f_{\boldsymbol{k}, \sigma} \varepsilon_{\boldsymbol{k}-\boldsymbol{q}}-\left\langle S_{z}\right\rangle \sum_{\sigma} \chi_{t}\left(\mathrm{i} q_{n}, \boldsymbol{q}, \sigma\right) .
$$

Thus, we completed the calculation of the spin propagator. Using the analytical continuation to the real frequency we found the spin wave energy

$$
\omega(\boldsymbol{q})=\omega_{0}-\frac{2\left\langle S_{z}\right\rangle}{N} \sum_{\boldsymbol{k}, \sigma} f_{\boldsymbol{k}, \sigma} \varepsilon_{\boldsymbol{k}-\boldsymbol{q}}-\left\langle S_{z}\right\rangle \sum_{\sigma} \chi_{t}(\omega, \boldsymbol{q}, \sigma) .
$$

$\omega(\boldsymbol{q})$ is gapless. At $\boldsymbol{q}=0$,

$$
\chi_{t}(\omega, 0, \sigma)=\frac{1}{N} \sum_{\boldsymbol{k}}\left(2 S \varepsilon_{\boldsymbol{k}}\right)^{2} \frac{f_{\boldsymbol{k}, \sigma}-f_{\boldsymbol{k},-\sigma}}{\omega-\varepsilon_{\boldsymbol{k}, \sigma}+\varepsilon_{\boldsymbol{k},-\sigma}} .
$$

In view of eqs. (10) and (11), we found a solution $\omega=0$ from eq. (33) by substituting eq. (34) into eq. (33). We evaluate the spin stiffness $\left(\omega=D_{S} \boldsymbol{q}^{2}\right)$ assuming cubic symmetry.

$$
\begin{aligned}
D_{S}= & -\sum_{\boldsymbol{k}, \sigma}\left(\frac{\left\langle S_{z}\right\rangle}{3}+\frac{S^{2}+\left\langle S_{z}\right\rangle^{2}}{12\left\langle S_{z}\right\rangle}-\frac{\sigma S}{2}\right) f_{\boldsymbol{k}, \sigma} \nabla^{2} \varepsilon_{\boldsymbol{k}} \\
& +\sum_{\boldsymbol{k}, \sigma} \frac{\left(S^{2}-\left\langle S_{z}\right\rangle^{2}\right)^{2}}{24 S\left\langle S_{z}\right\rangle^{2}} \sigma f_{\boldsymbol{k}, \sigma} \frac{\left(\boldsymbol{\nabla} \varepsilon_{\boldsymbol{k}}\right)^{2}}{\varepsilon_{\boldsymbol{k}}}
\end{aligned}
$$

It can easily derived that as $T \rightarrow 0,\left\langle S_{z}\right\rangle \rightarrow S, D_{S}$ is reduced to the form given by Kubo and Ohata, which was obtained with Holstein-Primikoff transformation. On the other hand, $D_{S} \rightarrow O\left(\left\langle S_{z}\right\rangle\right)$ as $\left\langle S_{z}\right\rangle \rightarrow 0$ and $T \rightarrow T_{\mathrm{C}}$ (see Appendix B).

\section{$\S 4 . \quad$ Results and Discussion}

The goal of this work is computing the spin stiffness. A by-product is that we calculated approximately the Curie temperature. Our calculation is self-consistent. For a given $\left\langle S_{z}\right\rangle$, we can adjust the chemical potential and the hole density so as to get the desired doping concentration. Now $\left\langle S_{z}\right\rangle$ can, in turn, be calculated from the ensemble of $H_{0}$. We can then adjust $\left\langle S_{z}\right\rangle$ to reach selfconsistency. At this stage, only $H_{0}$ is considered. We can do the computation in the whole temperature range. For a given doping concentration, we found that above certain temperature there is no solution for $\left\langle S_{z}\right\rangle \neq 0$. We deemed this temperautre $T_{\mathrm{C}}$.

The $T_{\mathrm{C}}$ from our calculation on double exchange (DE) alone is at about $800^{\circ} \mathrm{K}$ for $x=0.3$. It is three times of the experimental $T_{\mathrm{C}}$. However, there is superexchange interaction in manganites. To make our result conformable to realistic systems, we added a superexchange term (SE) to our Hamiltonian

$$
\frac{J}{2} \sum_{i, j} \boldsymbol{S}_{\boldsymbol{i}} \cdot \boldsymbol{S}_{\boldsymbol{j}}
$$

A reasonable value for $J$ is $t / 35$ where $t=0.2 \mathrm{eV}$ since the on-site Coulomb repulsion is around $U \approx 7 \mathrm{eV}$ and $J \approx t^{2} / U .^{26)}$ Since SE is quite small we can treated it with a mean-field approximation. It modifies $\omega_{0}$. Its contribution to $D_{S}$ was considered to the first order. The standard textbook approach gives the following modifi- cation:

$$
\begin{aligned}
\omega_{0} & \rightarrow \omega_{0}-z J\left\langle S_{z}\right\rangle \\
D_{S} & \rightarrow D_{S}-J a^{2}\left\langle S_{z}\right\rangle / 2
\end{aligned}
$$

where $z$ is the coordination number and $a$ is the lattice constant.

The results are shown in Figs. 1-3 in which crosses, the dashed lines and the solid lines represent those at doping $x=0.4,0.3$ and 0.2 respectively. In Fig. 1 we showed the averaged local spin. The upper (lower) set were calculated without (with) SE. Though it is not the magnetization, the curves showed approximately where $T_{\mathrm{C}}$ 's are. We have to be cautious because the results do not account for the fluctuations. They are self-consistent in the mean-field level. However, they give an approximate range of $T_{\mathrm{C}}$. It is lower than those given by eq. (4.12) of Kubo and Ohata ${ }^{11)}$ but are twice of the results of $\mathrm{Fu}-$ rukawa. ${ }^{23)}$ The fact that our result is not too far away from those of ref. 11 is expected though they used a different method to calculate $T_{\mathrm{C}}$ and their eq. (4.12) is an approximation. Furukawa used a infinite-dimension system and therefore, making comparison is difficult. We also noted that SE suppressed magnetization so that it was not saturated as $T \rightarrow 0$. This seems agree with experimental findings. Uhlenbruck et al. ${ }^{27)}$ had shown that the magnetic moments of manganese ions increase with applied magnetic field even at very low temperature.

SE reduced the $T_{\mathrm{C}}$ of $\mathrm{DE}$ by more than half even though its magnitude is rather small. The reason is that SE reduces $\left\langle S_{z}\right\rangle$. The change in $\left\langle S_{z}\right\rangle$ affects the shape of hole bands and change the numbers of spin-up and spindown holes. At finite temperature, it does not cost much energy because the holes near Fermi level can have their spins flipped. For the same reason, applying external magnetic field affects $T_{\mathrm{C}}$, moves it to the other direction. Hence, a little SE can have a large effect on the numbers of spin-up and spin-down holes, which in turn, are the deciding factors of the coupling between local spins. For the same reason large-doping materials are more susceptible to SE. The spins of holes are easier to flip if the chemical potential is higher. In Fig. 2 we show the influence of the superexchange interaction. $T_{\mathrm{C}}$ dereases as $J$ increases. For realistic ratio of $J / t \simeq 0.03, T_{\mathrm{C}}$ drops to $350^{\circ} \mathrm{K}$. Incorporating fluctuations will further suppress $T_{\mathrm{C}}$ and make it compatible with experimental results. We note here that we did not try to fit experimental $T_{\mathrm{C}}$. It is meaningless at this stage. Other effects such as the shapes of the bands, Jahn-Teller distortion and orbial degeneracies are not included in our calculation. Different ions, such as $\mathrm{La}^{+3}$ and $\mathrm{Ca}^{+2}$, giving different potentials will impede the motion of holes and diminish the coupling. If considered, $T_{\mathrm{C}}$ will be lower. Nevertheless, our $T_{\mathrm{C}}$ is quite close to experimental values. It would not have been the case if we, in view of eq. (1), had inferred that $T_{\mathrm{C}}$ is of the order $x z t /(2 S+1)$ due to the strength of spin waves. The spin stiffness softens as temperature rises. Thus, $T_{\mathrm{C}}$ is much lower than above prediction. One may understand it by the following arguement: as temperature rises, $\left\langle S_{z}\right\rangle$ becomes smaller. A smaller $\left\langle S_{z}\right\rangle$ means smaller energy difference between 


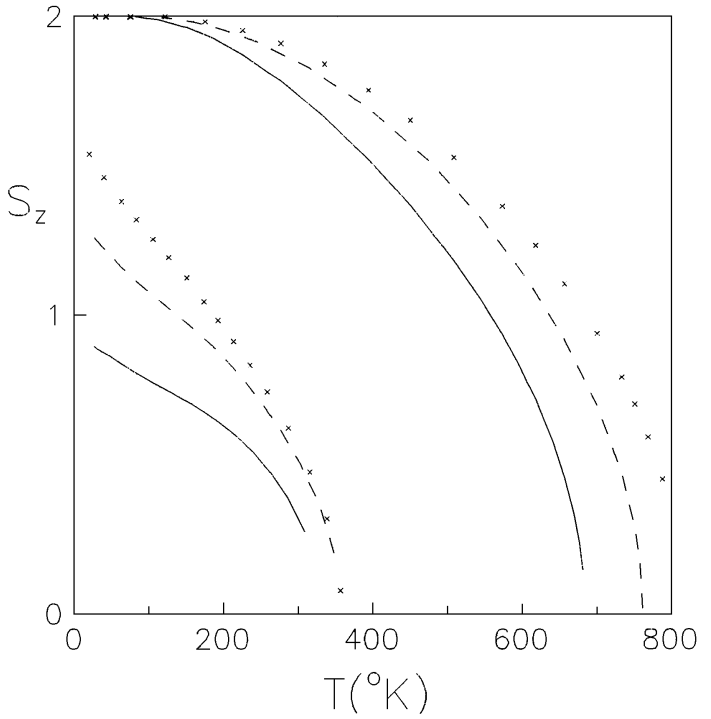

Fig. 1. Averaged local spin versus temperature. The results at doping $x=0.2,0.3$ and 0.4 are represented by the solid line, the dashed line and crosses. The lower set were computed with SE included.

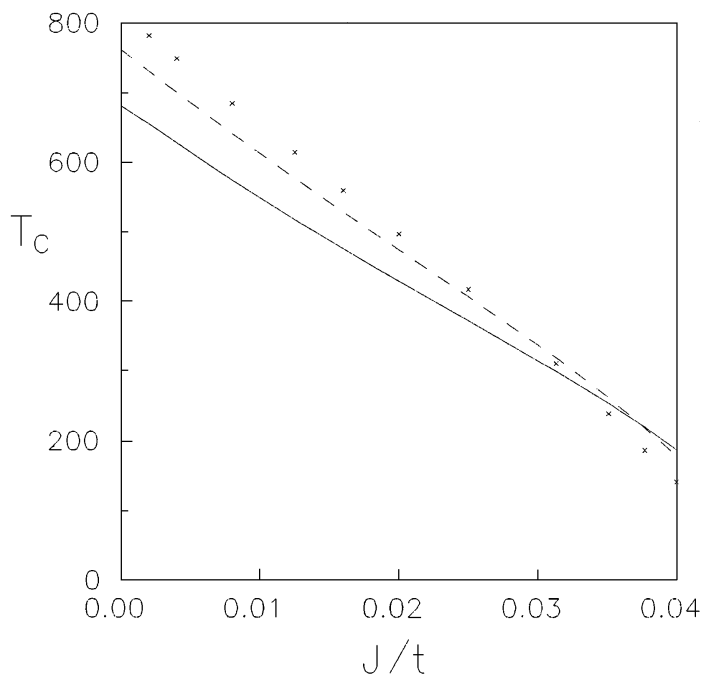

Fig. 2. $T_{\mathrm{C}}$ versus $J / t$. The results at doping $x=0.2,0.3$ and 0.4 are represented by the solid line, the dashed line and crosses.

spin-up and spin-down holes and smaller difference in abundances of spin-up and spin-down holes, which in turn, give even smaller difference in the energies of upand down- local spins and even smaller $\left\langle S_{z}\right\rangle$.

Recent numerical computations ${ }^{28-30}$ gave much lower $T_{\mathrm{C}}$, typically around $0.1 t$. This value has the same order of magnitude as experimental results. Our $T_{\mathrm{C}}$ is too high because it was calculated by mean-field approximation of DE. However, the fluctuation effect alone is unlikely to reduce $T_{\mathrm{C}}$ to such a low value. $\mathrm{SE}$ must play some role here. In ref. 30, Yi et al. showed that $T_{\mathrm{C}}$ was reduced by half if $\mathrm{SE}$ was introduced at the strength $J \simeq 0.3 t$. This is compatible with our calculation. This creats another

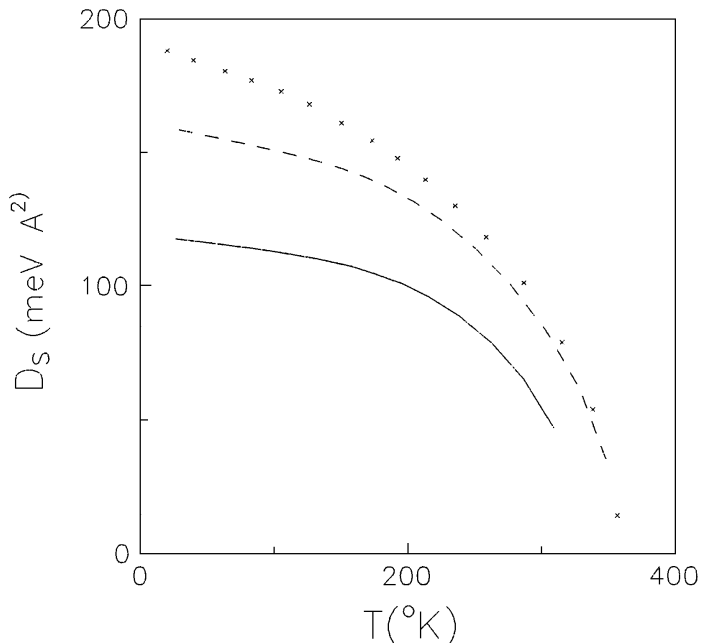

Fig. 3. $D_{S}$ versus temperature. The results at doping $x=0.2$, 0.3 and 0.4 are represented by the dashed line, the solid line and crosses.

problem. if $T_{\mathrm{C}}$ given by refs. $28-30$ is correct, adding $\mathrm{SE}$ which does exist in manganites, will make it too low. Apparently, more study is in order.

The experimental data on the spin waves are quite consistent. ${ }^{20,21)}$ The manganites have isotropic spin waves. There is no noticeable enegy gap. The spin wave stiffness $D_{S}$ is expected to increase with $x$. At $x=1 / 3$, $D_{S} \simeq 170 \mathrm{meVA}^{2}$ and decreases with increasing temperature. We show in Fig. $3 D_{S}$ versus $T$. From our calculation, $D_{S} \simeq 160 \mathrm{meVA}^{2}$ at $x=0.3$. Also, at $T / T_{\mathrm{C}}=3 / 5$, $D_{S}(T) / D_{S}(0) \simeq 4 / 5$ from experiments, similar in Fig. 3. We also noticed that there is significant fluctuation of yet unknown origin near $T_{\mathrm{C}}$. We did not attempt to make comparison since our calculation is not accurate in this region. By analyzing the DE Hamiltonian, it can be attributed the the fact $S_{z}$ is not a good quantum number. The quantum fluctuation becomes significant at $T \rightarrow T_{\mathrm{C}}$. It is possible that this is the cause for the deviation between our result and experimental data in the temperature dependence of $D_{S}$.

In conclusion, we have studied the DE Hamiltonian in details and found that we can perform the $1 /(2 S+1)$ expansion at $T<T_{\mathrm{C}}$. We then calculated the spin wave propagator at finite temperature. The resulting spin wave stiffness was in reasonably good agreement with experimental data. Adding superexchange interaction can reduce the Curie temperature to the range of real systems. We concluded that DE is the fundamental mechanism in perovskite manganites.

This work is supported in part by the National Science Council, Taiwan ROC under the contract number NSC 88-2112-M-002-007.

\section{Appendix A}

In this appedix, we give expressions of two propagators. 


$$
\begin{aligned}
\left\langle\widehat{T} S_{+}\right. & \left.(\tau) S_{-}\left(\tau^{\prime}\right) S_{z}\left(\tau_{1}\right) S_{z}\left(\tau_{2}\right)\right\rangle \\
= & -\left\langle S_{z}^{3}\right\rangle \mathcal{D}\left(\tau-\tau^{\prime}\right)+2\left\langle S_{z}^{2}\right\rangle \mathrm{e}^{\omega_{0}\left(\tau_{2}-\tau_{1}\right)}\left\{2 n_{B}\left[\xi\left(\tau^{\prime}, \tau_{1}, \tau_{2}, \tau\right)+\xi\left(\tau^{\prime}, \tau_{2}, \tau_{1}, \tau\right)\right]\right. \\
& -2\left(n_{B}+1\right)\left[\xi\left(\tau, \tau_{1}, \tau_{2}, \tau^{\prime}\right)+\xi\left(\tau, \tau_{2}, \tau_{1}, \tau^{\prime}\right)\right]+n_{B}\left[\xi\left(\tau^{\prime}, \tau_{1}, \tau, \tau_{2}\right)+\xi\left(\tau^{\prime}, \tau_{2}, \tau, \tau_{1}\right)+\xi\left(\tau_{1}, \tau^{\prime}, \tau_{2}, \tau\right)+\xi\left(\tau_{2}, \tau^{\prime}, \tau_{1}, \tau\right)\right] \\
& \left.-\left(n_{B}+1\right)\left[\xi\left(\tau, \tau_{1}, \tau^{\prime}, \tau_{2}\right)+\xi\left(\tau, \tau_{2}, \tau^{\prime}, \tau_{1}\right)+\xi\left(\tau_{1}, \tau, \tau_{2}, \tau^{\prime}\right)+\xi\left(\tau_{2}, \tau, \tau_{1}, \tau^{\prime}\right)\right]\right\} \\
& +2\left\langle S_{z}\right\rangle\left\{2 n_{B}\left(n_{B}+1\right)\left[\left(n_{B}+1\right) \theta\left(\tau-\tau^{\prime}\right)+n_{B} \theta\left(\tau^{\prime}-\tau\right)\right]+\left(n_{B}+1\right)^{2} \theta\left(\tau-\tau^{\prime}\right)\right. \\
& -n_{B}^{2} \theta\left(\tau^{\prime}-\tau\right)+n_{B}\left(n_{B}+1\right)\left[\xi\left(\tau^{\prime}, \tau, \tau_{1}, \tau_{2}\right)+\xi\left(\tau^{\prime}, \tau, \tau_{2}, \tau_{1}\right)+\xi\left(\tau_{1}, \tau^{\prime}, \tau, \tau_{2}\right)\right. \\
& +\xi\left(\tau_{2}, \tau^{\prime}, \tau, \tau_{1}\right)+\xi\left(\tau_{1}, \tau_{2}, \tau^{\prime}, \tau\right)+\xi\left(\tau_{2}, \tau_{1}, \tau^{\prime}, \tau\right)-\xi\left(\tau, \tau^{\prime}, \tau_{1}, \tau_{2}\right) \\
& \left.\left.-\xi\left(\tau, \tau^{\prime}, \tau_{2}, \tau_{1}\right)-\xi\left(\tau_{1}, \tau, \tau^{\prime}, \tau_{2}\right)-\xi\left(\tau_{2}, \tau, \tau^{\prime}, \tau_{1}\right)-\xi\left(\tau_{1}, \tau_{2}, \tau, \tau^{\prime}\right)-\xi\left(\tau_{2}, \tau_{1}, \tau, \tau^{\prime}\right)\right]\right\}
\end{aligned}
$$

Its form in frequency representation is less lengthy. However, we will show the Fourier transformed expression after it was multiplied by a term $(1 / \beta) \sum_{l} F\left(\mathrm{i} p_{l}\right) \mathrm{e}^{\mathrm{i} p_{l}\left(\tau_{1}-\tau_{2}\right)}$. It will be useful in evaluating eq. (27).

$$
\begin{aligned}
& -\frac{1}{2 \beta} \sum_{l} \int_{-\beta}^{\beta} \mathrm{d}\left(\tau-\tau^{\prime}\right) \mathrm{e}^{\mathrm{i} p_{l}\left(\tau-\tau^{\prime}\right)} \int_{0}^{\beta} \mathrm{d} \tau_{1} \int_{0}^{\beta} \mathrm{d} \tau_{2} \mathrm{e}^{\mathrm{i} p_{l}\left(\tau_{1}-\tau_{2}\right)} F\left(\mathrm{i} p_{l}\right)\left\langle\widehat{T} S_{+}(\tau) S_{-}\left(\tau^{\prime}\right) S_{z}\left(\tau_{1}\right) S_{z}\left(\tau_{2}\right)\right\rangle \\
& =\sum_{l} F\left(\mathrm{i} p_{l}\right)\left\{\delta_{0 p_{l}}\left[\frac{2 \beta\left\langle S_{z}^{3}\right\rangle}{\mathrm{i} q_{n}-\omega_{0}}+\frac{4\left\langle S_{z}^{2}\right\rangle}{\left(\mathrm{i} q_{n}-\omega_{0}\right)^{2}}+n_{B}\left(n_{B}+1\right) \frac{4 \beta\left\langle S_{z}\right\rangle}{\left(\mathrm{i} q_{n}-\omega_{0}\right)^{2}}\right]\right. \\
& \left.\quad+\frac{2 \beta\left\langle S_{z}\right\rangle}{p_{l}}\left[\frac{2}{\mathrm{i} q_{n}-\omega_{0}}-\frac{1}{\mathrm{i} q_{n}+\mathrm{i} p_{l}-\omega_{0}}-\frac{1}{\mathrm{i} q_{n}-\mathrm{i} p_{l}-\omega_{0}}\right]\right\}
\end{aligned}
$$

Last term gives a disconnected diagram, so it will be canceled. We also have

$$
\begin{aligned}
\left\langle\widehat{T} S_{+}\right. & \left.(\tau) S_{-}\left(\tau^{\prime}\right) S_{+}\left(\tau_{1}\right) S_{-}\left(\tau_{2}\right)\right\rangle \\
= & \left\langle S_{z}^{2}\right\rangle\left[\mathcal{D}\left(\tau-\tau_{2}\right) \mathcal{D}\left(\tau_{1}-\tau^{\prime}\right)+\mathcal{D}\left(\tau-\tau^{\prime}\right) \mathcal{D}\left(\tau_{1}-\tau_{2}\right)\right] \\
& -2\left\langle S_{z}\right\rangle\left\{\left(n_{B}+1 / 2\right)\left[\mathcal{D}\left(\tau-\tau_{2}\right) \mathcal{D}\left(\tau_{1}-\tau^{\prime}\right)+\mathcal{D}\left(\tau-\tau^{\prime}\right) \mathcal{D}\left(\tau_{1}-\tau_{2}\right)\right]\right. \\
& +\left(n_{B}+1\right)\left[\xi\left(\tau, \tau^{\prime}, \tau_{1}, \tau_{2}\right)+\xi\left(\tau, \tau_{2}, \tau_{1}, \tau^{\prime}\right)+\xi\left(\tau_{1}, \tau^{\prime}, \tau, \tau_{2}\right)+\xi\left(\tau_{1}, \tau_{2}, \tau, \tau^{\prime}\right)\right] \\
& \left.+n_{B}\left[\xi\left(\tau^{\prime}, \tau, \tau_{2}, \tau_{1}\right)+\xi\left(\tau^{\prime}, \tau_{1}, \tau_{2}, \tau\right)+\xi\left(\tau_{2}, \tau, \tau^{\prime}, \tau_{1}\right)+\xi\left(\tau_{2}, \tau_{1}, \tau^{\prime}, \tau\right)\right]\right\} .
\end{aligned}
$$

The terms of the order $\left\langle S_{z}\right\rangle$ will be neglected. For the two terms of the order $\left\langle S_{z}\right\rangle^{2}$, the second one gives a disconnected diagam. Hence, taking the Fourier transform of the first term and the contribution from the holes, we obtain eq. (28).

\section{Appendix B}

To see the behavior of $D_{S}$ as $\left\langle S_{z}\right\rangle \rightarrow 0$, we note first that

$$
f_{\boldsymbol{k},-}-f_{\boldsymbol{k},+}=4 S\left\langle S_{z}\right\rangle \varepsilon_{\boldsymbol{k}} \frac{\partial f_{\boldsymbol{k}, 0}}{\partial \varepsilon_{\boldsymbol{k}, 0}}+O\left(S_{z}^{3}\right),
$$

where

$$
\varepsilon_{\boldsymbol{k}, 0}=\frac{S^{2} \varepsilon_{\boldsymbol{k}}}{(2 S+1)^{2}}
$$

and $f_{\boldsymbol{k}, 0}$ is the Fermi function with energy $\varepsilon_{\boldsymbol{k}, 0}$. Substituting eq. (B.1) into eq. (33) and integrating by parts, we found that $D_{S}=O\left(S_{z}\right)$.

1) G. H. Jonker and J. H. Santen: Physics 16 (1950) 337.

2) E. O. Wollen and W. C. Koehler: Phys. Rev. 100 (1955) 548.

3) For a recent review on experimental reuslts, see A. P. Ramirez: J. Phys. C: Condens. Matter 9 (1997) 8171.

4) C. Zener: Phys. Rev. 82 (1951) 403.

5) A. J. Millis, P. B. Littlewood and B. I. Shraiman: Phys. Rev. Lett. 74 (1995) 5144; J. Millis, Boris I. Shraiman and R.
Mueller: Phys. Rev. Lett. 77 (1996) 175.

6) J.-S. Zhou and J. B. Goodenough: Phys. Rev. Lett. 80 (1998) 2665 .

7) T. T. M. Palstra et al.: Phys. Rev. B 56 (1997) 5104.

8) J. P. Franck, I. Isaac, Weimin Chen, J. Chrzanowski and J. C. Irwin: Phys. Rev. B 58 (1998) 5189.

9) P. W. Anderson and Hasegawa: Phys. Rev. 100 (1955) 675.

10) De Gennes: Phys. Rev. 118 (1960) 141.

11) K. Kubo and N. Ohata: J. Phys. Soc. Jpn. 33 (1972) 21.

12) Nobuo Furukawa: J. Phys. Soc. Jpn. 63 (1994) 3214.

13) L. Sheng, H. Y. Tong, D. N. sheng and C. S. Ting: Phys. Rev. B 58 (1997) 8186.

14) D. I. Glosov, M. R. Norman and K. Levin: Phys. Rev. B 58 (1998) 8617.

15) Daniel P. Arovas and Francisco Guinea: Phys. Rev. B 58 (1998) 9150.

16) Ryo Maezono, Sumio Ishihara and Naoto Nagaosa: Phys. Rev. B 58 (1998) 11583.

17) D. M. Edwards, A. C. M. Green and K. Kubo: J. Phys. Condens. Matt. 11 (1999) 2791.

18) Sanjoy K. Sarkar: J. Phys.: Condens. Matt. 8 (1996) L515.

19) Erwin Müller-Hartmann and Elbio Dagotto: Phys. Rev. B 54 (1996) R6819.

20) J. A. Fernandez-Baca, P. Dai, H. Y. Hwang, C. Kloc and S.-W. Cheong: Phys. Rev. Lett. 80 (1998) 4012.

21) J. W. Lynn, R. W. Erwin, J. A. Borchers, Q. Huang and A. Santoro: Phys. Rev. Lett. 76 (1996) 4046.

22) W. E. Pickett and D. J. Singh: Phys. Rev. B 53 (1996) 1146; D. J. Singh and W. E. Pickett: Phys. Rev. B 57 (1998) 88.

23) Nobuo Furukawa: J. Phys. Soc. Jpn. 65 (1996) 1174.

24) Similar but different computation can be found in B. Giovannini, M. Peter and S. Koide: Phys. Rev. 149 (1965) 
251; Bernard Giovannini and Shoichiro Koide: Prog. Theo. Phys. 34 (1965) 705

25) M Gaudin: Nucl. Phys. 15 (1960) 89.

26) L. Sheng, D. N. Sheng and C. S. Ting: Phys. Rev. 59 (1999) 13550 .
27) S. Uhlenbruck et al.: Phys. Rev. Lett. 82 (1999) 185.

28) H. Röder et al.: Phys. Rev. B 56 (1997) 5084.

29) S. Yunoki et al.: Phys. Rev. Lett. 80 (1998) 845.

30) Hongsuk Yi, Jaejun Yu and Sung-Ik Lee: arXiv: condmat/9910152 (1999). 